\title{
Geometric sensitivity of beacon placement using airborne mobile anchors
}

\author{
Izanoordina Ahmad \\ Electronics Section, Universiti Kuala Lumpur-British Malaysian Institute, Malaysia
}

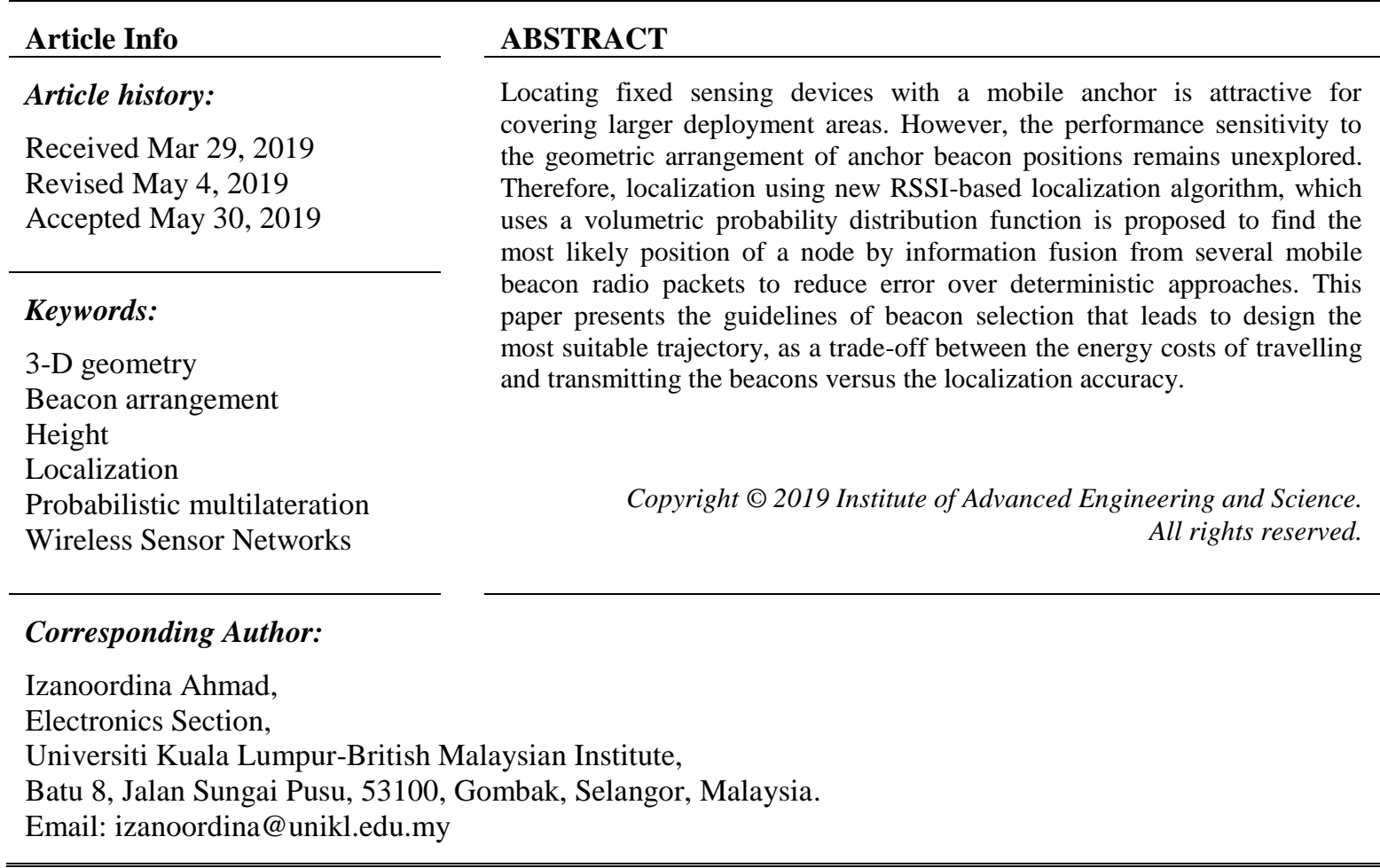

\section{INTRODUCTION}

Localization is important for many Wireless Sensor Networks (WSN) applications. When a sensor is deployed, its sensor data is often of limited use unless the position of the sensor is known when the measurement was taken. While technologies like GPS are now relatively cheap, the additional circuitry, antennas, energy use and computational resources are not always suitable for low cost, low-energy sensors, especially where the sensor is static and only needs to be localised once. Additionally, GPS is not always available due to occlusion by buildings, trees or other obstructions. For this reason, there is keen interest in other methods for localizing sensors. This paper considers a motivating scenario where the sensor nodes are carried by an aircraft and are then dropped and randomly scattered within the sensing region such as in the application of bushfire monitoring [1]. In this scenario, these nodes are not guaranteed to land at particular locations or in particular orientations. The nodes should be lightweight and rugged enough to minimize the possibility of being damaged during their deployment [2]. Localization can be achieved by using the same aircraft to act as a mobile anchor. The aircraft can be equipped with GPS and can broadcast its position at regular intervals along a specific trajectory. The deployed sensor nodes are blind nodes [3].

One of the main issues is whether the mobile anchor's trajectory may influence the localization performance depending on the number of beacons sent and the positions of the mobile anchor nodes. Therefore, we will investigate two sets of parameters for a commonly used flight path trajectory, double square grid that will be analysed to determine the path length. Another parameter is to determine the effects of varying the height of beacons and the distance or spaces between beacons on localization error. Multilateration is known to be significantly affected by anchor geometry. For mobile anchors, the position of beacons depends on the flight path, and the spacing of beacon messages in that path. There are limited studies 
on mobile anchor placement since more attention has been given to localization accuracy and computational effort. Guidelines to optimize the beacon placement by considering the ratio between the distance and the radio range, and the minimum height of the triangle formed by the anchors and the blind node is proposed in [4]. The impact of anchor node placement and its rules as well as using the smallest number of anchor nodes are discussed. This work is in 2D scenario but it can be expanded to 3D localization. Some aspects of beacon positioning especially the effect of anchor node placement on the localization errors on a network-wide basis is explored in [5]. This approach could minimize the number of anchors required while avoiding poor localization.

In $[6,7]$, three beacon points from among the received beacons are selected and the intersection area with two beacon points are obtained to calculate the location of the blind node using the third beacon point to reduce the ambiguity of the intersection area. They assumed straight line movement of a mobile beacon at a constant speed. The communication range between mobile anchor node and the unknown sensor is 20 metres and the mobile anchor node will broadcasts a beacon every 1 metre. However this algorithm is applied to 2D localization and there is a possibility of having a flip ambiguity due to the straight movement. Flip ambiguity is a phenomenon in localization caused by inappropriate geometric relations between the anchors [8]. In 2D localization, anchors which are co-linear or close to co-linear will have a region where the multilateration least square error objective function is small close to the actual location of the blind node. In [9], the analysis of flip ambiguity considered the ranging errors caused by the environmental noise. They proposed an algorithm to detect and solve the flip ambiguity problem in various situations. While their work is focused on the flip ambiguities in trilateration, it also can be extended to a multilateration algorithm. Flip ambiguity is a particular problem with airborne anchors, since a naïve flight path, which flies over an area at a constant height is exactly the situation that can cause flip ambiguity. Thus, in my work on path planning, this problem will need to be considered.

An important issue in using mobile anchor nodes will be what path the mobile anchor node should follow, when and where it should transmit beacon packets. More beacon packets can potentially improve localization accuracy, but it will have effects on the time and energy needed for localization. Path planning may be pre-planned, or it may react to information from blind-nodes (e.g. some node may indicate they have insufficient packets for accurate positioning, so the mobile anchor node may change its path to accommodate these nodes). The algorithm for anchor placement can be categorized as random, statically planned or dynamically planned trajectories [10-12]. Poorly planned trajectories may cause a large localization delay, low localization ratio and increase the localization error. The statically planned trajectory is a well-planned path designed which should provide non-coplanar anchor positions to avoid the flip ambiguity issues. The localization using a static trajectory is further discussed in [13]. However, most of the positioning schemes are based on a range-free approach that is not part of our research. Static path planning schemes such as those in [14-19] consider factors such as the mobile anchor node movement strategy, number of mobile anchor nodes, number of transmitted message for localization and the comparison between choices of trajectory pattern.

Localization performance [20-23] can be evaluated through performance metrics such as localization accuracy, computational complexity, energy efficiency, time taken, number of anchors to be deployed and communication overhead. Motivated by this factor, we will investigate the localization error based on the beacon placement that consists of height of beacon and the beacon spacing using proposed algorithms. These guidelines can lead us to design a beacon path, which will give suitable beacon geometry for the scattered blind nodes. One possible approach is to use Gradient Descent so that beacon positions to move to positions that give better and better accuracy, and then draw conclusions about the geometrical features of good beacon arrangements.

\section{RESEARCH METHOD}

A multilateration algorithm and optimization of the number of anchors using Deterministic Multilateration (DML) and Volume based Probabilistic Multilateration (VPML) algorithms have been analysed in [3, 24, 25]. However, there is limited analysis of geometric sensitivity of beacon positions and mobile anchor trajectories. Thus, further research and experiments will be discussed in this section. In order to design the best flight path of the aircraft and to calculate the best beacon placement along that flight path, this research aims to find good mobile anchor node positions for multiple blind node localization on the ground. Positioning involves tension between having high radio signal strength, which gives lower ranging uncertainty and between having spaced out anchors. These experiments also investigate how accuracy varies across the sensing region. Furthermore, the height and the spacing between beacons and path length will be determined for the best compromise between path length and accuracy. 
Therefore, the number of simulation iterations that are needed to give consistent estimates of performance is investigated. In our previous experiment [3] 100 iterations were used, but these experiments are more complex (potentially thousands of beacons, tens of blind nodes), and so a smaller number of iterations is helpful to reduce the experimental run time if it still gives consistent results. The chosen flight path is a square 2D grid pattern. To avoid flip ambiguity, the patterns must include measurements at different heights, giving the double square grid pattern. The experiments here use a number of blind nodes spread through the sensing region, including some in unfavourable positions near the region edge. The experiments will investigate not just the average accuracy, but also how accuracy varies across the sensing region. These experiments use a probabilistic simulation environment (VPML) as shown in Figure 1 due to its performance that gives better error results for beacon localization than DML. The possible height of beacons and the spacing between beacons will be determined by comparing different combinations of aircraft height and beacon spacing. For a large number of beacons, the highest signal strength beacons may all be close together, which is not a good geometry.

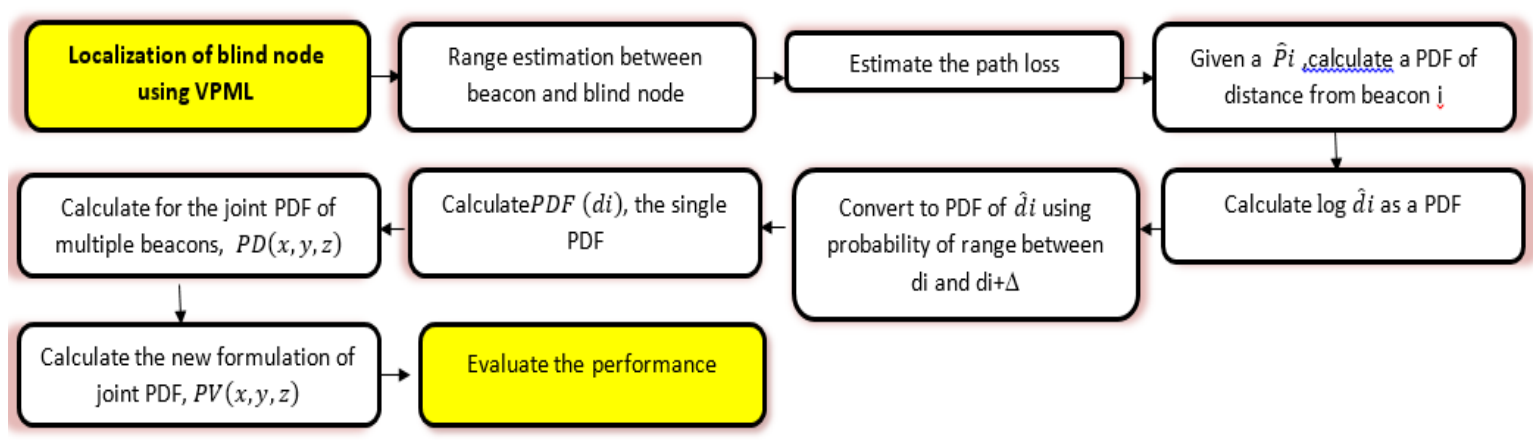

Figure 1. Block diagram of VPML

\subsection{Volume based Probabilistic Multilateration Localization (VPML)}

Given a path loss $\hat{P} i$, a PDF of possible distances from beacon $i(d i)$ can be calculated;

$$
\hat{P} i=P o+10 n[\log \hat{d} i-\log d o]+\hat{r} \sigma p
$$

Note that $P o$ is the path loss at $d o$ which is $28.43 \mathrm{~dB}, n$ is the $\log$ normal shadowing power at 3.3647. While $\hat{r}$ is the zero mean Gaussian random variable with standard deviation of path loss $\sigma p$ at $3.37 \mathrm{~dB}$. Assuming the reference distance used for the experimental measurement, do is 1 metre, then $\log d o=0$.

$$
\log \hat{d} i=\frac{\hat{P} i-P o}{10 n}-\frac{\hat{r} \sigma p}{10 n}
$$

Thus $\log \hat{d} i$ can be represented as a Probability Distribution Function (PDF) of RSSI:

$$
\begin{aligned}
& \log \hat{d} i=\frac{P i-P o}{10 n}-\hat{r} \sigma d \\
& \text { where, } \sigma d=\frac{\sigma p}{10 n}
\end{aligned}
$$

The (3) gives a PDF of the likelihood of a particular log-distance for the range, given a path loss value. Therefore, we need to convert to a PDF of distance, not log-distance. To convert to a PDF of $\hat{d} i$, we add a correction factor. It must be true that the probability of the real range being between di and di $+\Delta$ must be the same in both cases.

$$
P D F(\log d i) \cdot[\log (\hat{d} i+\Delta)-\log (\hat{d} i)]=P D F(d i) x(\hat{d} i+\Delta-d i)
$$




$$
\begin{aligned}
& P D F(d i)=\frac{\log (\hat{d} i+\Delta)-\log (\hat{d} i)]}{\Delta} P D F \log d i=\frac{d}{\hat{d} i}(\log \hat{d} i) P D F \log d i \\
& =\frac{1}{\hat{d} i} P D F \log d i
\end{aligned}
$$

PDF of $\log d i$ is a Gaussian with mean $(\mu)=\frac{P i-P o}{10 n}$ and standard deviation $(\sigma)$ of $\sigma d$. Using (6), we can define;

$$
P D F(\log d i)=\frac{1}{\sigma \sqrt{2 \pi}} e^{-\frac{(\log \hat{d} i-\mu)^{2}}{2 \sigma^{2}}}
$$

Thus,

$$
P D F(d i)=\frac{1}{\widehat{d} i \sigma \sqrt{2 \pi}} e^{-\frac{(\log \hat{d} i-\mu)^{2}}{2 \sigma^{2}}}
$$

For the multiple beacons, the probability that the blind node is $\hat{d} i$ away from beacon $i$ for all $i$, is conventionally calculated using the product of the PDFs. Here, we calculate the joint PDF based on the product of individual PDFs as in (7). The probability of being at a certain position is the probability of being a certain range from beacon 1 and being a certain range from beacon 2, etc. Thus;

$$
P D(x, y, z)=\prod_{i} P D F i(x, y, z)
$$

where PDFi is the PDF of being distance $d$ from beacon $i$ while PD is the un-normalized joint PDF that the blind node is a certain distance from beacon 1 and a certain distance from beacon 2 , until all distances from each beacon $i$ are estimated.

The actual PDF would require the PDF to be divided by the volume integral of the function above to normalize the total likelihood of being anywhere in the volume to 1 . However, we are only looking for the maximum value of the joint PDF. The maximum of the un-normalized joint PDF will be at the same location as a PDF normalized over the whole volume. An optimization approach can then be used to find the point where $P D(x, y, z)$ is a maximum, and this is the estimated position. This is the conventional approach to probabilistic localization, as described in [26]. This conventional approach, however, can be improved by closer examination of the geometry.

In the above formulation, each PDF is a function of a single variable, distance. Given that we are searching for the best point in a 3-D space, it could also be argued that we should use a PDF based on volume, not distance. Given a $\operatorname{PDF}(\mathrm{d})$ based on distance from a beacon, the $\operatorname{PDF}(\mathrm{x}, \mathrm{y}, \mathrm{z})$ that the blind node at a particular point at that range can be calculated. A point at a range of $\mathrm{d}$ in the one-dimensional PDF corresponds to the surface of a sphere, radius $d$, centred at the beacon. The probability that the blind node is in an infinitesimal interval $d+\Delta d$ in the 1-D PDF corresponds to the probability that the blind node is in a spherical shell, inner radius $\mathrm{d}$, outer radius $\mathrm{d}+\Delta \mathrm{d}$. The volume of the shell is surface area times thickness, $4 \pi \mathrm{d}^{2} \Delta \mathrm{d}$. Since this shell volume increases with $\mathrm{d}^{2}$, the volumetric PDF in 3-D will also scale with $\mathrm{d}^{2}$ compared to the linear PDF. In particular, the volumetric PDF at a particular point on that sphere is $\frac{P D F i(d)}{4 \pi d^{2} \Delta d}$ Again, ignoring constants $(4 \pi)$ and $\Delta \mathrm{d}$, this gives a relative volumetric PDF for one beacon range of:

$$
P D F V i=\frac{P D F i}{d i^{2}}
$$

Then a new joint PDF for being at a certain point based on all the range estimates is:

$$
P V(x, y, z)=\prod_{i} \frac{P D F i(x, y, z)}{d i^{2}}
$$

In other words, we further scale each PDF by a factor of $\mathrm{di}^{2}$ before multiplying them together. Here, we investigated which of $P D(x, y, z)$ and $P V(x, y, z)$ works best. In both cases, we used simple gradient descent convex function optimization to find the maximum value of the joint PDF, starting from the DML estimated position. This gives the $(x, y, z)$ which maximizes $P D$ or $P V$. Overall, his paper explores the geometrical sensitivity and the airborne trajectory using the examination of VPML. 


\subsection{Simulation setup}

Four experiments will be conducted where the real distance is used with a random number generator to give an estimated path loss between beacons and blind nodes. Any path loss that exceeds the sensitivity of $-90.5 \mathrm{~dB}$ will be discarded. Then, a maximum of 20 of the strongest beacon packets will be used to observe the relationship between number of anchor positions versus localization error. Finally, the estimated position of the blind nodes using VPML will be determined. The parameters used in this simulation are;

a. $\quad$ Spacing is the distance between the beacons in the $\mathrm{X}$ and $\mathrm{Y}$ direction.

b. Height is the $\mathrm{Z}$ position of the beacon.

Experiment 1: This experiment is to determine the number of iteration for multiple blind node localization at various geometrical position using VPML algorithm. The objective is to identify the number of simulation iterations to reduce the computation time but maintain stable statistics using the VPML algorithm only. The simulation is run using the following number of iterations $(5,10,20,30,40,50,60,70,80,90$ and 100) at 10 and 13 metre heights. These numbers of iterations are chosen to accurately identify where median error stabilizes. The proposed square grid path planning is used to localize a single blind node at 500,500,0 actual position. The median error versus number of iterations will be plotted to determine the point of stable statistics.

Experiment 2: This experiment is to determine the possible height of beacons. The square grid path is designed to send a beacon at every position with a particular X-Y spacing and with alternate rows at different heights to localize a blind node. Two nodes at 500,500,0 (directly under a beacon) and 127,192,0 (between beacons) are localized. The objective is to determine which are the best heights for alternate rows of beacons in the square grid flightpath. The heights of the mobile anchor (consisting of alternate lower and upper paths) is initially tested using a range of height combinations such as 10 and 13 metres, 10 and 15 metres, 20 and 23 metres and 20 and 25 metres. These combinations are based on the estimated height above the obstacles with localization error which is less than 30 metres (considered as extremely high error). Note that 10 metres is chosen as a minimum height to avoid obstacles such as trees. Solutions will use the best $\mathrm{N}$ nodes, where $\mathrm{N}$ is varied from 4 to 20 . This experiment will also justify the combination of heights that will be used for future simulations.

Experiment 3: The third experiment is to determine the possible spacing between beacons. This experiment is to compare the localization performance at different beacon spacing by using square grid spacing of 5,10 , 20, 30, 40, 50 and 60 metres with alternate heights determined by the results from the previous section. The maximum spacing of $60 \mathrm{~m}$ is chosen since this is approximately the maximum radio range for a receiver sensitivity of $-90.5 \mathrm{~dB}$. In this simulation, a square grid path with these different spacing and heights is used to localize one blind node located centrally $(500,500,0)$ within a $1 \mathrm{~km} \times 1 \mathrm{~km}$ area using VPML with up to 20 beacon packets. Examples of a square grid path with alternate heights and different beacon spacing are shown in Figure 2.

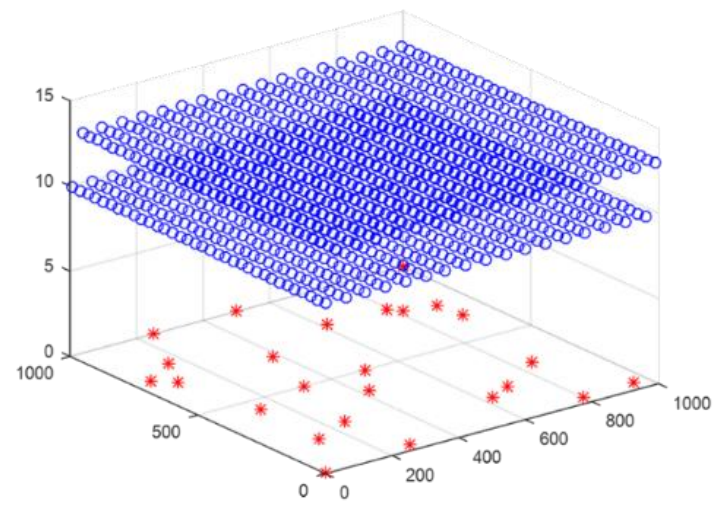

Figure 2. Square grid path with $30 \mathrm{~m}$ beacon spacing and alternate layers of $10 \mathrm{~m}$ and $13 \mathrm{~m}$ height

Experiment 4: This experiment is to determine the path length and number of beacons using the proposed square grid path. Using the square grid path planning with the best alternate heights from the previous experiment, the various localization error for 25 blind nodes at various positions. The number of transmitted 
beacons, minimum and maximum number of received beacons and length of path can be retrieved. The maximum number of beacons is expected to vary depending on the location of the blind node. The aim of this experiment is to compare the factors, which determine the time and energy needed by the mobile anchor to traverse the grid. These factors are travel distance and number of transmitted beacons. Grid spacing of $5 \mathrm{~m}$, $10 \mathrm{~m}, 20 \mathrm{~m}$ and $30 \mathrm{~m}$ are used, with alternate heights of $10 \mathrm{~m} \& 13 \mathrm{~m}$.

\section{RESULTS AND ANALYSIS}

\subsection{Number of iteration for multiple blind node localization}

The result is based on an experiment using a square grid path for localizing a favourable blind node at 500,500,0. Using a spacing of 10 metres between beacons and alternate 10 and 13 metres height, the localization errors versus number of iterations are plotted for 5 different runs. So, for, say, 10 iterations, the median accuracy over 10 simulation runs is calculated and plotted and this is repeated 5 times. The five different median values are examined. If there are insufficient iterations, each trial will give significantly different results. For sufficient iterations, the individual medians should be tightly clustered. The results are plotted in Figure 3. From these results, it can be seen that for 40 or more iterations, the results are tightly clustered from the 5 trials. The standard deviation in Table 1 is relatively low from 40 to 100 iterations with the lowest at 70 . Therefore, it can be suggested that 70 iterations is the best choice to provide a good balance between computational efficiency and error consistency across the trials. Thus, for the rest of these experiments, it will be used to calculate median localization errors.

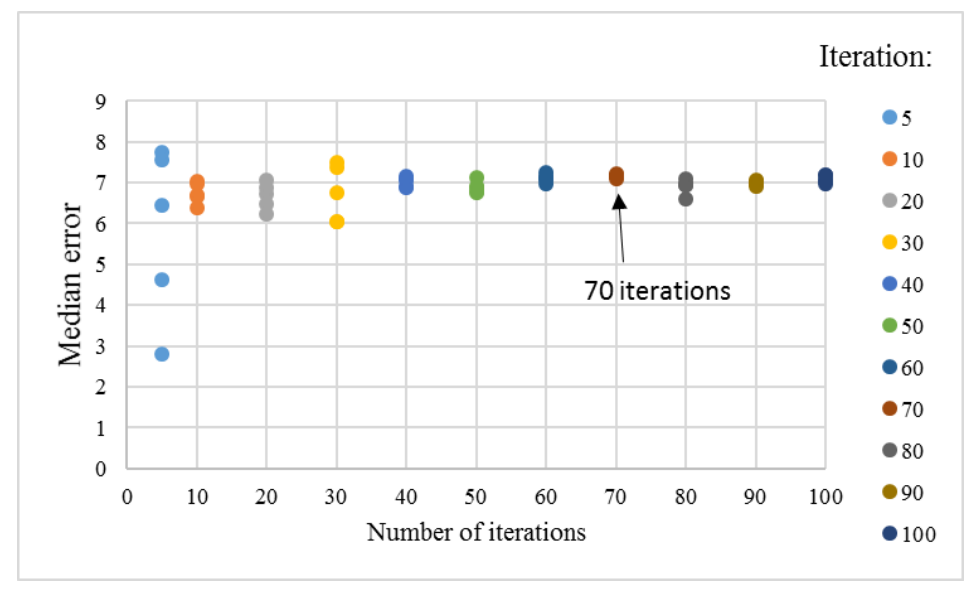

Figure 3. Median error (m) versus number of iterations for 5 trials

Table 1. Standard deviation for each of 5 trials

\begin{tabular}{cccccccccccc}
\hline Trial & \multicolumn{10}{c}{ Number of iterations } \\
\hline & 5 & 10 & 20 & 30 & 40 & 50 & 60 & 70 & 80 & 90 & 100 \\
1 & 2.81 & 6.97 & 7.07 & 7.50 & 6.92 & 6.74 & 7.11 & 7.10 & 6.94 & 7.06 & 7.07 \\
2 & 4.62 & 6.68 & 6.49 & 6.05 & 7.03 & 6.90 & 7.08 & 7.22 & 6.61 & 6.99 & 7.18 \\
3 & 6.45 & 6.68 & 6.22 & 6.06 & 7.16 & 6.79 & 6.99 & 7.19 & 6.94 & 7.02 & 7.04 \\
4 & 7.74 & 7.05 & 6.73 & 7.36 & 7.10 & 7.13 & 7.15 & 7.17 & 7.09 & 6.90 & 6.98 \\
5 & 7.57 & 6.40 & 6.88 & 6.74 & 6.87 & 6.90 & 7.24 & 7.14 & 6.96 & 6.99 & 7.20 \\
Standard deviation & 2.10 & 0.26 & 0.33 & 0.69 & 0.13 & 0.15 & 0.09 & 0.04 & 0.18 & 0.06 & 0.09 \\
\hline
\end{tabular}

\subsection{Possible height of beacons}

The result for experiment 2 is to determine the best possible combination height for alternate rows of beacons in the square grid flightpath as shown in Figure 4 for the two nodes. This shows that the performance is significantly better for the $10 / 13 \mathrm{~m}$ and $10 / 15 \mathrm{~m}$ alternate heights for both nodes. It also shows that error reduces as more beacons are used up to about 13 nodes, and does not significantly improve beyond that. The next experiment will investigate the best heights using a lower height of $10 \mathrm{~m}$ and an upper height of $11-15 \mathrm{~m}$. 

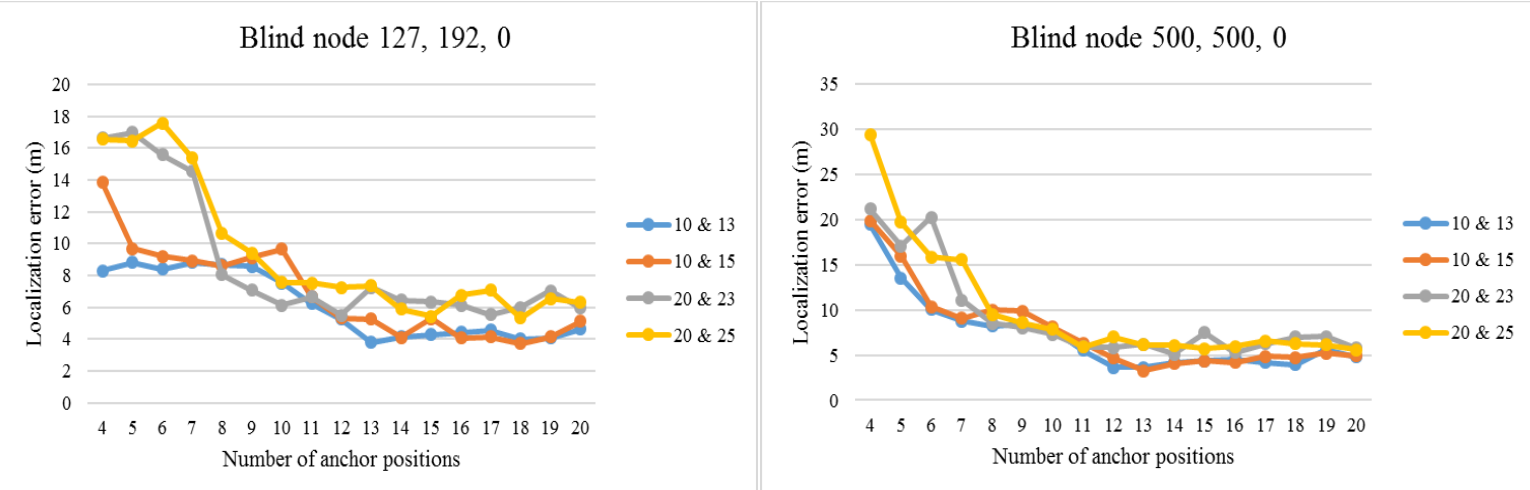

Figure 4. Comparison between height for blind node 127,192,0 and 500,500,0

\subsection{Possible spacing between beacons}

Experiment 3 investigated whether a blind node could reliably receive 20 beacon messages, and if so what the localization error was, using the different grid spacing of 5, 10, 20,30,40,50, and 60m and different heights. Based on results in Figure 5, it was found that for $20 \mathrm{~m}$ spacing and less, 20 beacons could reliably be received. For $30 \mathrm{~m}$, less than 20 beacons were received, but localization could still be achieved. The previous experiments showed that around 12 or 13 beacons are needed for good localization. For $40 \mathrm{~m}$ and beyond, the blind node had insufficient beacons to give good localization. In terms of path heights, the combination of 10 and $13 \mathrm{~m}$ gave the best results for $5 \mathrm{~m}$ and $20 \mathrm{~m}$ spacing and was very close to the best for $10 \mathrm{~m}$ spacing. Therefore, for future experiments, grid spacing up to $30 \mathrm{~m}$ will be examined, and heights of $10 \mathrm{~m}$ and $13 \mathrm{~m}$ will be used.

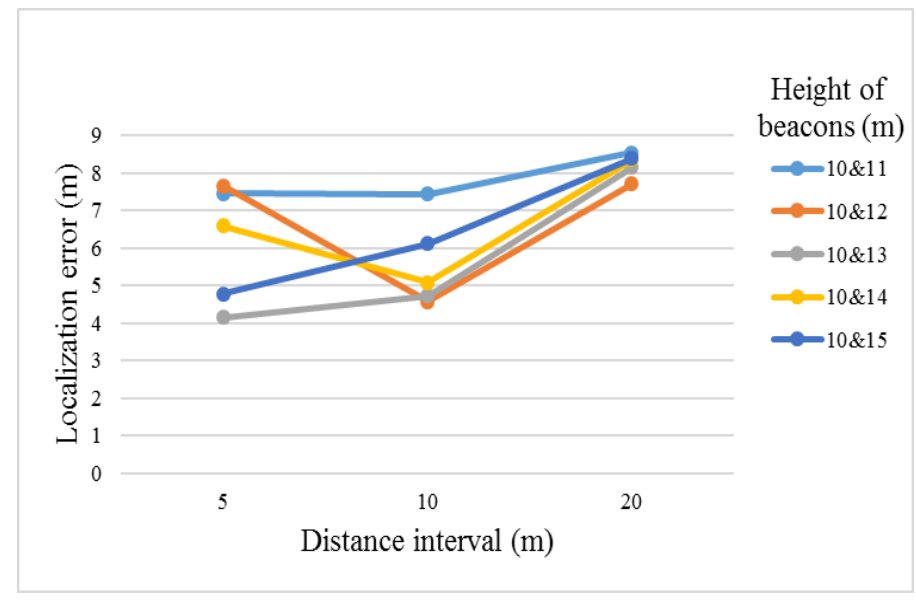

Figure 5. Comparison between localization errors versus beacon distance interval using 20 beacons

\subsection{Path length and number of beacons}

The following results Figures 6 (a) (b) and (c) show the localization error for 3 blind nodes at different positions. The three nodes are located at favourable $(500,500,0)$, unfavourable $(0,0,0)$ and between-beacon positions $(142,439,0)$. The result is generated based on localization using a minimum of 4 to a maximum of 20 anchor positions for square grid path planning using 70 simulation iterations.

The result for blind node 500,500,0 in Figure 5 shows that with spacing of $5 \mathrm{~m}$ and $10 \mathrm{~m}$, the localization error reduces until about 14 beacons, and then it is relatively constant. However, the errors for $20 \mathrm{~m}$ spacing increase after 8 beacons and are relatively constant for $30 \mathrm{~m}$ spacing up to the maximum available beacons. Blind node 0,0,0 could only be localized using $5 \mathrm{~m}$ and $10 \mathrm{~m}$ spacing with 20 beacon positions. For $20 \mathrm{~m}$ and $30 \mathrm{~m}$ spacing, error is large. The result of blind node $142,439,0$ shows $20 \mathrm{~m}$ spacing gives lowest error up to about 11 beacons, however the $20 \mathrm{~m}$ error increases starting from 12 beacon positions. $5 \mathrm{~m}$ and $10 \mathrm{~m}$ spacing continue to improve with more beacons. Based on these results, $5 \mathrm{~m}$ and $10 \mathrm{~m}$ 
spacing give significantly better accuracy than $20 \mathrm{~m}$ and $30 \mathrm{~m}$ spacing, provided that sufficient beacons (14 or more) are used. For most blind nodes, $20 \mathrm{~m}$ spacing still gives 20 available nodes, however for nodes near the edge this number falls significantly, and is sometimes as low as 10 , again suggesting $20 \mathrm{~m}$ spacing is just at the edge of reliable localization, and would only be recommended if the mobile anchor path length was a major factor.

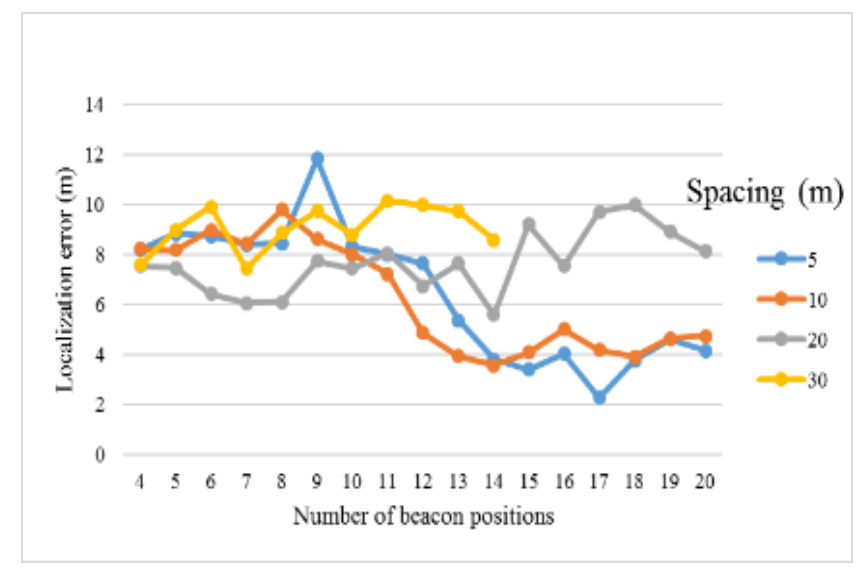

(a)

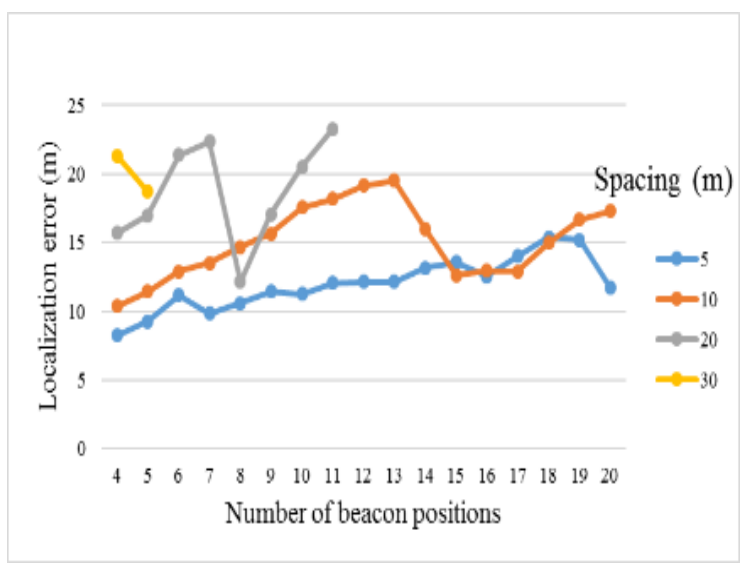

(b)

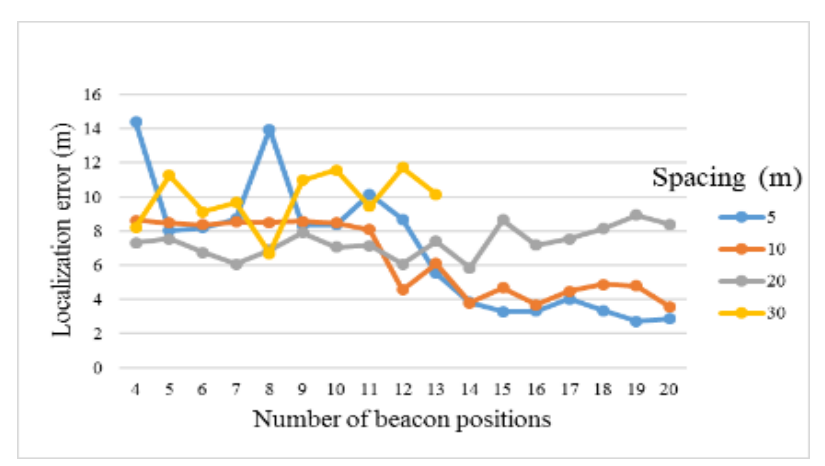

(c)

Figure 6. Average localization error for blind node, (a) $(500,500,0),(b)(0,0,0)$, and (c) $(142,439,0)$

However, there is not a clear advantage in using $5 \mathrm{~m}$ spacing, and in some cases, it gives poorer results than $10 \mathrm{~m}$ spacing. As can be seen from Table 2, it requires twice the travel distance and 4 times as many radio transmissions, with negligible improvement in accuracy. Therefore, for this particular scenario, a $10 \mathrm{~m}$ spacing is the preferred option. These experimental results are specific to this scenario, but they do demonstrate that localization of air-dropped sensors using an airborne mobile anchor is feasible, and that simulation experiments can suggest suitable operational parameters for that localization. Thus, for scenario with different parameter values, a set of similar experiments as in this section could be conducted.

Table 2. Path characteristics for different grid spacing

\begin{tabular}{ccccc}
\hline \multirow{2}{*}{ Characteristics } & \multicolumn{4}{c}{ Spacing } \\
& $5 \mathrm{~m}$ & $10 \mathrm{~m}$ & $20 \mathrm{~m}$ & $30 \mathrm{~m}$ \\
\hline Maximum Beacons & 20 & 20 & 20 & 14 \\
Minimum Beacons & 20 & 20 & 10 & 0 \\
Transmitted & 40200 & 10201 & 2601 & 1156 \\
Length of Path & $201 \mathrm{~km}$ & $102 \mathrm{~km}$ & $52 \mathrm{~km}$ & $35 \mathrm{~km}$ \\
\hline
\end{tabular}




\section{CONCLUSION}

These experiments aim to address the trade-off between the energy costs of travelling and transmitting the beacons versus the accuracy of the localization, as well as choosing a good path geometry. The results here are not universal results, and they do not give a flight path that is suitable for all sensor deployments. What they do is describe a methodology by which such decisions can be made through simulation of a specific scenario. Firstly, a square grid with alternate height rows has been shown to give good localization which avoids flip ambiguity. A square grid is suitable for a square search area compared to some of the other possible paths described in section 2, and it is the only geometry considered in detail here. Different generic flightpaths could be more appropriate for different shaped deployment areas. Secondly, simulation of different alternate heights can be used to determine suitable heights which minimize errors. In this case $10 \mathrm{~m}$ and $13 \mathrm{~m}$ were identified as best.

Thirdly, an initial investigation of possible grid spacing can eliminate those options where there are often insufficient beacons to localize nodes well. In this scenario, spacing more than $30 \mathrm{~m}$ did not give reliable localization and were not investigated further. For the best quality of localization results, up to 20 beacons can be used to improve localization performance. Fourthly, different grid spacing can be simulated to find the best spacing, which trades off accuracy for flight time and the number of transmitted beacons. In my scenario, a spacing of $10 \mathrm{~m}$ was identified as the best compromise. For scenarios with different radio ranges, the compromise may be different. It was observed that one node, right at the corner of the sensing area $(1000,1000,0)$ could not reliably receive enough beacons to be localized. This suggests that a flight path will need to extend slightly beyond the limits of the deployment area to enable all nodes to be localized. Finally, the results obtained for geometric sensitivity showed that it is not always best to choose the strongest beacons to use for localization. Investigating the best way to select beacons based on the relative angles between rays from the 20 closest beacons is suggested as useful future work.

\section{REFERENCES}

[1] T. Wark et al., "Springbrook: Challenges in developing a long-term, rainforest wireless sensor network," 2008 International Conference on Intelligent Sensors, Sensor Networks and Information Processing, Sydney, NSW, 2008, pp. 599-604.

[2] P. Pounds, T. Potie, F. Kendoul, S. Singh, R. Jurdak, and J. Roberts, "Automatic distribution of disposable self-deploying sensor modules," in Experimental Robotics, pp. 535-543. 2016.

[3] I. Ahmad, N. W. Bergmann, R. Jurdak and B. Kusy, "Towards probabilistic localization using airborne mobile anchors," 2016 IEEE International Conference on Pervasive Computing and Communication Workshops (PerCom Workshops), Sydney, NSW, 2016, pp. 1-4.

[4] N. A. Alwan and A. S. Mahmood, "Distributed gradient descent localization in wireless sensor networks," Arabian Journal for Science and Engineering, vol. 40, no. 3, pp. 893-899, 2015.

[5] B. Tatham and T. Kunz, "Anchor node placement for localization in wireless sensor networks," 2011 IEEE 7th International Conference on Wireless and Mobile Computing, Networking and Communications (WiMob), Wuhan, 2011, pp. 180-187.

[6] R. Akl, K. Pasupathy and M. Haidar, "Anchor nodes placement for effective passive localization," 2011 International Conference on Selected Topics in Mobile and Wireless Networking (iCOST), Shanghai, 2011, pp. 127-132.

[7] S. Lee, E. Kim, C. Kim and K. Kim, "Localization with a mobile beacon based on geometric constraints in wireless sensor networks," in IEEE Transactions on Wireless Communications, vol. 8, no. 12, pp. 5801-5805, December 2009.

[8] P. Moravek, D. Komosny, M. Simek, and J. Muller, "Multilateration and flip ambiguity mitigation in ad-hoc networks," Przeglad Elektrotechniczny (Electrical Review), vol. 88, no. 5b, pp. 222-229, 2012.

[9] S. Han, J. Yue, W. Meng and C. Li, "A Novel Flip Ambiguities Detection Algorithm for WSN Localization," 2015 IEEE Globecom Workshops (GC Wkshps), San Diego, CA, 2015, pp. 1-6.

[10] O. Artemenko, A. Rubina, T. Simon, and A. Mitschele-Thiel, "Evaluation of different static trajectories for the localization of users in a mixed indoor-outdoor scenario using a real unmanned aerial vehicle," in International Conference on Ad Hoc Networks, pp. 123-133: Springer. 2015,

[11] Kuo-Feng Ssu, Chia-Ho Ou and H. C. Jiau, "Localization with mobile anchor points in wireless sensor networks," in IEEE Transactions on Vehicular Technology, vol. 54, no. 3, pp. 1187-1197, May 2005.

[12] X. Li, N. Mitton, I. Simplot-Ryl and D. Simplot-Ryl, "Dynamic Beacon Mobility Scheduling for Sensor Localization," in IEEE Transactions on Parallel and Distributed Systems, vol. 23, no. 8, pp. 1439-1452, Aug. 2012.

[13] Y. Yun, J. Jin, N. Kim, J. Yoon and C. Kim, "Outdoor localization with optical navigation sensor, IMU and GPS," 2012 IEEE International Conference on Multisensor Fusion and Integration for Intelligent Systems (MFI), Hamburg, 2012, pp. 377-382.

[14] Hongjun Li, Jianwen Wang, Xun Li and Hongxu Ma, "Real-time path planning of mobile anchor node in localization for wireless sensor networks," 2008 International Conference on Information and Automation, Changsha, 2008, pp. 384-389. 
[15] S. M. Mazinani and F. Farnia, "Localization in wireless sensor network using a mobile anchor in obstacle environment," International Journal of Computer and Communication Engineering, vol. 2, no. 4, p. 438, 2013.

[16] C. Ou and W. He, "Path Planning Algorithm for Mobile Anchor-Based Localization in Wireless Sensor Networks," in IEEE Sensors Journal, vol. 13, no. 2, pp. 466-475, Feb. 2013.

[17] R. Huang and G. V. Zaruba, "Static Path Planning for Mobile Beacons to Localize Sensor Networks," Fifth Annual IEEE International Conference on Pervasive Computing and Communications Workshops (PerComW'07), White Plains, NY, 2007, pp. 323-330.

[18] K. Kim and W. Lee, "MBAL: A Mobile Beacon-Assisted Localization Scheme for Wireless Sensor Networks," 2007 16th International Conference on Computer Communications and Networks, Honolulu, HI, 2007, pp. 57-62.

[19] G. Han, C. Zhang, J. Lloret, L. Shu, and J. J. Rodrigues, "A mobile anchor assisted localization algorithm based on regular hexagon in wireless sensor networks," Scientific World Journal, vol. 2014, pp. 1-13, 2014.

[20] T. Chai and R. R. Draxler, "Root mean square error (RMSE) or mean absolute error (MAE)? - arguments against avoiding RMSE in the literature," Geoscientific Model Development, vol. 7, no. 3, pp. 1247-1250, 2014.

[21] Y. Sabri and N. El Kamoun, "A distributed method to localization for mobile sensor networks based on the convex hull," International Journal of Advanced Computer Science and Applications (IJACSA) vol. 3, pp. 1-9, 2012.

[22] C. Miao, G. Dai, K. Ying, and Q. Chen, "Collaborative localization and location verification in WSNs," Sensors, vol. 15, no. 5, pp. 10631-49, May 062015.

[23] R. Jurdak et al., "Camazotz: multimodal activity-based GPS sampling," in Proceedings of the 12th International Conference on Information Processing in Sensor Networks, pp. 67-78: ACM. 2013.

[24] I. Ahmad, N. Bergmann, R. Jurdak and B. Kusy, "Experiments on localization of wireless sensors using airborne mobile anchors," 2015 IEEE Conference on Wireless Sensors (ICWiSe), Melaka, 2015, pp. 1-6.

[25] B. Dawes and K.-W. Chin, "A comparison of deterministic and probabilistic methods for indoor localization," Journal of Systems and Software, vol. 84, no. 3, pp. 442-451, 2011.

[26] R. Peng and M. L. Sichitiu, "Probabilistic localization for outdoor wireless sensor networks," ACM SIGMOBILE Mobile Computing and Communications Review, vol. 11, no. 1, pp. 53-64, 2007.

\section{BIOGRAPHY OF AUTHOR}

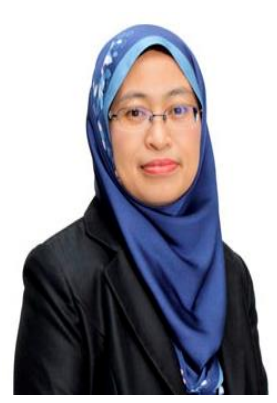

Izanoordina Ahmad is a senior lecturer of Electronics section at Universiti Kuala Lumpur- British Malaysian where she has been a faculty member since 2000. Izanoordina completed her Ph.D. at University of Queensland, Australia and her undergraduate studies at Leeds Metropolitan University in United Kingdom. Her research interests lie in the area of Electrical and Electronics Engineering, ranging from the theory to design to implementation. She has collaborated actively with researchers in several other disciplines of computer science and telecommunications particularly in Internet of Things on problems at the hardware and software interface. 